\title{
$G_{2 n}$ SPACES( $\left.{ }^{1}\right)$
}

BY

\section{DONALD O. KOEHLER}

\begin{abstract}
A complex normed linear space $X$ will be called a $G_{2 n}$ space if and only if there is a mapping $\langle\cdot, \ldots, \cdot\rangle$ from $X^{2 n}$ into the complex numbers such that: $x_{k} \rightarrow\left\langle x_{1}, \ldots, x_{2 n}\right\rangle$ is linear for $k=1, \ldots, n ;\left\langle x_{1}, \ldots, x_{2 n}\right\rangle=\left\langle x_{2 n}, \ldots, x_{1}\right\rangle^{-}$; and $\langle x, \ldots, x\rangle^{1 / 2 n}=\|x\|$. The basic models are the $L^{2 n}$ spaces, but one also has that every inner product space is a $G_{2 n}$ space for every integer $n$. Hence $G_{2 n}$ spaces of a given cardinality need not be isometrically isomorphic. It is shown that a complex normed linear space is a $G_{2 n}$ space if and only if the norm satisfies a generalized parallelogram law. From the proof of this characterization it follows that a linear map $U$ from $X$ to $X$ is an isometry if and only if $\left\langle U\left(x_{1}\right), \ldots, U\left(x_{2 n}\right)\right\rangle=\left\langle x_{1}, \ldots, x_{2 n}\right\rangle$ for all $x_{1}, \ldots, x_{2 n}$. This then provides a way to construct all of the isometries of a finite dimensional $G_{2 n}$ space. Of particular interest are the CBS $G_{2 n}$ spaces in which $\left|\left\langle x_{1}, \ldots, x_{2 n}\right\rangle\right| \leqq\left\|x_{1}\right\|$ $\cdots\left\|x_{2 n}\right\|$. These spaces have many properties similar to inner product spaces. An operator $A$ on a complete CBS $G_{2 n}$ space is said to be symmetric if and only if $\left\langle x_{1}, \ldots, A\left(x_{i}\right), \ldots, x_{2 n}\right\rangle=\left\langle x_{1}, \ldots, A\left(x_{j}\right), \ldots, x_{2 n}\right\rangle$ for all $i$ and $j$. It is easy to show that these operators are scalar and that on $L^{2 n}, n>1$, they characterize multiplication by a real $L^{\infty}$ function. The interest in nontrivial symmetric operators is that they exist if and only if the space can be decomposed into the direct sum of nontrivial $G_{2 n}$ spaces.
\end{abstract}

1. Introduction. The purpose of this paper is to study normed linear spaces in which the norms arise from multilinear forms in much the same way that the norm of an inner product space arises from an inner product. The motivation for this study comes from considering $X=L^{2 n}(Y, \Sigma, \mu)$ where $(Y, \Sigma, \mu)$ is a measure space. Analogous to the case when $n=1$, for $x_{1}, \ldots, x_{2 n}$ in $X$ we define

$$
\left\langle x_{1}, \ldots, x_{2 n}\right\rangle=\int x_{1} \cdots x_{n} \bar{x}_{n+1} \cdots \bar{x}_{2 n} d \mu
$$

Presented to the Society, August 29, 1969; received by the editors October 22, 1969.

AMS Subject Classifications. Primary 4620; Secondary 4635, 4730, 4740.

Key Words and Phrases. Generalized-inner-products, semi-inner-products, inner products, norms from multilinear forms, generalized polarization identity, generalized parallelogram law, $G_{2 n}$ spaces, CBS $G_{2 n}$ spaces, isometries on $G_{2 n}$ spaces, symmetric operators on CBS $G_{2 n}$ spaces.

( $\left.{ }^{1}\right)$ Part of the research was performed while the author was at the U.S.A.F. Aerospace Research Laboratories while in the capacity of an Ohio State University Research Foundation Visiting Research Associate under Contract F33615 67 C 1758.

This paper is a revised version of a portion of the author's Ph.D dissertation (March 1968) from Indiana University, Bloomington, Indiana. The author wishes to express his sincere thanks to Professor Louis Brickman, who directed the dissertation, and to Professor Robert Bonic, who originally suggested the problem. 
where - denotes complex conjugate. We then have:

(i) $\|x\|^{2 n}=\langle x, \ldots, x\rangle$.

(ii) $x_{k} \rightarrow\left\langle x_{1}, \ldots, x_{2 n}\right\rangle$ is linear for $k=1, \ldots, n$ and conjugate linear for $k=n+1, \ldots, 2 n$.

(iii) $\left\langle x_{2 n}, \ldots, x_{1}\right\rangle=\left\langle x_{1}, \ldots, x_{2 n}\right\rangle^{-}$.

The similarity with an inner product suggests the following definition.

Definition (1.2). Let $X$ be a complex normed linear space. We shall say that $X$ is a $G_{2 n}$ space if and only if there is a mapping from $X^{2 n}$ into the complex numbers satisfying (i)-(iii) above. Such a mapping is called a generalized-inner-product (of order $2 n$ ). This will be abbreviated by g.i.p.

To simplify the notation we shall observe several notational conventions. We shall replace $\left\langle x_{1}, \ldots, x_{2 n}\right\rangle$ by $x_{1} \cdots x_{2 n}$ and use exponents to indicate the repeated occurrence of a given vector. For example

$$
x y^{n-1} x y^{n-1}=\langle x, y, \ldots, y, x, y, \ldots, y\rangle
$$

where $y$ appears in each of the $n-1$ positions following an $x$. Also, $x^{2 n}=\|x\|^{2 n}$ and $\left(x^{2 n}\right)^{1 / 2 n}=\|x\|$. We shall sometimes find it convenient to use two sets of $n$ vectors, $x_{1}, \ldots, x_{n}, y_{1}, \ldots, y_{n}$, and shall write $\mathbf{x y}$ for $x_{1} \cdots x_{n} y_{1} \cdots y_{n}$. Finally, if $\sigma$ and $\rho$ are permutations of $\{1, \ldots, n\}$ we shall write $\sigma(\mathbf{x}) \rho(\mathbf{y})$ for

$$
x_{\sigma(1)} \cdots x_{\sigma(n)} y_{\rho(1)} \cdots y_{\rho(n)} .
$$

If we are given any g.i.p. we can define

$$
[\mathbf{x}, \mathbf{y}]=(n !)^{-2} \sum_{\sigma, \rho} \sigma(\mathbf{x}) \rho(\mathbf{y})
$$

where the sum is to be taken over all $(n !)^{2}$ pairs of permutation $\sigma, \rho$ of $\{1, \ldots, n\}$. (1.3) then defines a g.i.p. with the added property.

$$
[\tau(\mathbf{x}), \pi(\mathbf{y})]=[\mathbf{x}, \mathbf{y}]
$$

for all permutations $\tau$ and $\pi$ of $\{1, \ldots, n\}$. We shall assume for the rest of the paper that all g.i.p.'s have this property.

We shall first consider some examples to illustrate the variety of these spaces.

EXAMPLE (1.4). Let $X$ be an abstract complex inner product space with inner product $(\cdot, \cdot)$. Let

$$
\mathbf{x y}=(n !)^{-1} \sum_{\sigma}\left(x_{1}, y_{\sigma(1)}\right) \cdots\left(x_{n}, y_{\sigma(n)}\right)
$$

where the summation is taken over all permutations $\sigma$ of $\{1, \ldots, n\}$. Then $X$ is a $G_{2 n}$ space with the inner product space norm.

This example, along with the $L^{2 n}$ example, shows that $G_{2 n}$ spaces of a given cardinality need not be isometrically is'omorphic.

EXAMPLE (1.6). Let $X$ be a finite dimensional complex vector space of dimension $r$, and let $m \geqq r$. Let $f_{1}, \ldots, f_{m}$ be any $m$ linear functionals on $X$ such that any $r$ 
of them are linealy independent. (The fact that it is possible to pick these functionals can be established by induction on $m$.) Let

$$
\mathbf{x y}=\sum_{j} f_{j}\left(x_{1}\right) \cdots f_{j}\left(x_{n}\right) f_{j}\left(y_{1}\right)^{-} \cdots f_{j}\left(y_{n}\right)^{-} .
$$

If we let

$$
\|x\|=\left(x^{2 n}\right)^{1 / 2 n}=\left(\sum_{j}\left|f_{j}(x)\right|^{2 n}\right)^{1 / 2 n},
$$

the fact that each $f_{j}$ is linear, together with the Minkowski inequality, helps show that (1.8) defines a norm.

LEMMA (1.9). If $m \geqq 2 r-1$ and $n \geqq 2$ then $X$, with the norm generated by (1.7), is not (isometrically isomorphic to) $l_{2 n, r}\left(r\right.$ dimensional $l^{2 n}$ ).

Proof. If $0=x y^{n-1} x y^{n-1}=\sum\left|f_{j}(x)\right|^{2}\left|f_{j}(y)\right|^{2 n-2}$, then for $j=1, \ldots, 2 r-1$, either $f_{j}(x)=0$ or $f_{j}(y)=0$. This means that at least one of $x$ and $y$ is in the kernel of at least $r$ linearly independent linear functionals, and must be 0 (the zero vector in $X$ ). On the other hand $l_{2 n, r}$ has a norm generated by

$$
\mathbf{z w}=\sum z_{1 j} \cdots z_{n j} \bar{w}_{1 j} \cdots \bar{w}_{n j} .
$$

If we let $x=(1,0, \ldots, 0)$ and $y=(0,1,0, \ldots, 0)$ then $x y^{n-1} x y^{n-1}=0$ while neither $x$ nor $y$ is 0 . We shall see in Corollary (2.4) that any linear isometry between $G_{2 n}$ spaces must preserve the g.i.p.'s. As a result, $X$, with the norm generated by (1.7), is not isometrically isomorphic to $l_{2 n, r}$.

EXAMPLE (1.9). If $X$ and $Y$ are $G_{2 n}$ spaces with g.i.p.'s $\langle\cdot, \ldots, \cdot\rangle_{1}$ and $\langle\cdot, \ldots, \cdot\rangle_{2}$ then their direct sum is a $G_{2 n}$ space with the g.i.p. defined by

$$
\left\langle\left(x_{1}, y_{1}\right), \ldots,\left(x_{2 n}, y_{2 n}\right)\right\rangle=\left\langle x_{1}, \ldots, x_{2 n}\right\rangle_{1}+\left\langle y_{1}, \ldots, y_{2 n}\right\rangle_{2} .
$$

We shall return to this example in $\$ 5$ when we consider the relationship between the decomposibility of a $G_{2 n}$ space into the direct sum of nontrivial $G_{2 n}$ spaces and the existence of "symmetric" operators. This problem is related to the problem of the classification of $G_{2 n}$ spaces which appears to be a difficult problem.

2. The polarization and related identities. In the course of developing the theory of inner product spaces one is led to consider relationships between the inner product and the associated quadratic form. Among these are the polarization identity, the parallelogram law, and the Jordan-von Neumann theorem which states that the norm arises from an inner product if and only if it satisfies the parallelogram law. These relationships are concerned with identities of the form

$$
\sum_{k} \lambda_{k}\left\|\sum_{j} a_{k j} x_{j}\right\|^{2}=f\left(x_{1}, \ldots\right)
$$

For example, the polarization identity is

$$
\sum_{k=0}^{3} i^{k}\left\|x+i^{k} y\right\|^{2}=4(x, y) .
$$


The parallelogram law,

$$
\|x+y\|^{2}+\|x-y\|^{2}-2\|x\|^{2}-2\|y\|^{2}=0,
$$

is also of this form.

In the $G_{2 n}$ spaces we shall be considering generalizations of these ideas, and hence must consider expressions of the form $\sum_{k} \lambda_{k}\left\|\sum_{j} a_{k j} x_{j}\right\|^{2 n}$. As an example we consider the "polarization identity for $G_{2 n}$ spaces."

THEOREM (2.1). In any $G_{2 n}$ space

$$
\sum_{\mathbf{h}, \mathbf{k}} i^{|\mathbf{h}|}\|F(\mathbf{x}, \mathbf{h}, \mathbf{k})\|^{2 n}=4^{2 n}(n !)^{2} x_{1} \cdots x_{2 n}
$$

where

$$
F(\mathbf{x}, \mathbf{h}, \mathbf{k})=i^{k_{1}} x_{1}+\cdots+i^{k_{n}} x_{n}+i^{k_{1}+h_{1}} x_{n+1}+\cdots+i^{k_{n}+h_{n}} x_{2 n},
$$

and where the summation is taken over all possible n-vectors $\mathbf{h}$ and $\mathbf{k}$ whose components are nonnegative integers between 0 and $3,|\mathbf{h}|=h_{1}+\cdots+h_{n}$.

The proofs of this theorem and the next will be a little easier if we first consider the evaluation of $\left\|\sum a_{j} x_{j}\right\|^{2 n}$. This will be a little easier if we think of it in terms of evaluating the corresponding expression of complex numbers

$$
\left|\sum a_{j} x_{j}\right|^{2 n}=\left(\sum a_{j} x_{j}\right)^{n}\left[\left(\sum a_{j} x_{j}\right)^{n}\right]^{-},
$$

and realize that most of the computations are the parallel of the computations in the corresponding multinomial expansions.

Thus

$$
\left\|\sum_{j=1}^{p} a_{j} x_{j}\right\|^{2 n}=\sum_{\mathbf{r}, \mathbf{s}} C_{n, \mathbf{r}} C_{n, \mathbf{s}}\left(a_{1} x_{1}\right)^{r_{1}} \cdots\left(a_{p} x_{p}\right)^{r_{p}}\left(a_{1} x_{1}\right)^{s_{1}} \cdots\left(a_{p} x_{p}\right)^{s_{p}}
$$

(where $\mathbf{r}=\left(r_{1}, \ldots, r_{p}\right)$ consists of nonnegative integers whose sum is $n$, and similarly for $\mathbf{s} . C_{n, \mathbf{r}}=n ! /\left(r_{1} ! \cdots r_{p} !\right)$ is the coefficient in the multinomial expansion, and the sum is to be taken over all possible vectors $\mathbf{r}$ and $\mathbf{s})$.

$$
=\sum_{\mathbf{r}, \mathbf{s}} c(\mathbf{a}, \mathbf{r}, \mathbf{s}) x_{1}^{r_{1}} \cdots x_{p}^{r_{p}} x_{1}^{s_{1}} \cdots x_{p}^{s_{p}}
$$

(where $c(\mathbf{a}, \mathbf{r}, \mathbf{s})=C_{n, \mathbf{r}} C_{n, \mathbf{s}} a_{1}^{r_{1}} \cdots a_{p}^{r_{p}} \bar{a}_{1}^{s_{1}} \cdots \bar{a}_{p}^{s_{p}}$, and - denotes complex conjugate).

$$
=\sum_{\mathbf{r}, \mathbf{s}} c(\mathbf{a}, \mathbf{r}, \mathbf{s}) d(\mathbf{x}, \mathbf{r}, \mathbf{s})
$$

(where $\left.d(\mathbf{x}, \mathbf{r}, \mathbf{s})=x_{1}^{r_{1}} \cdots x_{p}^{r_{p}} x_{1}^{s_{1}} \cdots x_{p}^{s_{p}}\right)$.

Proof of Theorem (2.1).

$$
\begin{aligned}
\sum_{\mathbf{h}, \mathbf{k}} i^{|\mathbf{h}|}\|F(\mathbf{x}, \mathbf{h}, \mathbf{k})\|^{2 n} & =\sum_{\mathbf{h}, \mathbf{k}} i^{|\mathbf{h}|} \sum_{\mathbf{r}, \mathbf{s}} c(a(\mathbf{h}, \mathbf{k}), \mathbf{r}, \mathbf{s}) d(\mathbf{x}, \mathbf{r}, \mathbf{s}) \\
& =\sum_{\mathbf{r}, \mathbf{s}}\left(\sum_{\mathbf{h}, \mathbf{k}} i^{|\mathbf{h}|} c(a(\mathbf{h}, \mathbf{k}), \mathbf{r}, \mathbf{s})\right) d(\mathbf{x}, \mathbf{r}, \mathbf{s}) .
\end{aligned}
$$


But for each $\mathbf{r}$ and $\mathbf{s}$

$$
\sum_{\mathbf{h}, \mathbf{k}} i^{|\mathbf{h}|} c(a(\mathbf{h}, \mathbf{k}), \mathbf{r}, \mathbf{s})=\sum_{\mathbf{h}, \mathbf{k}} C_{n, \mathbf{r}} C_{n, \mathbf{s}} i^{a_{1} k_{1}+\cdots+a_{n} k_{n}+b_{1} h_{1}+\cdots+b_{n} h_{n}}
$$

where $a_{j}=r_{j}+r_{n+j}-s_{j}-s_{n+j}$, and $b_{j}=1+r_{n+j}-s_{n+j}$ for $j=1, \ldots, n$. But this sum is 0 unless $a_{j} \equiv b_{j} \equiv 0(\bmod 4)$ for $j=1, \ldots, n$. And this is equivalent to

$$
s_{n+j}-r_{n+j} \equiv r_{j}-s_{j} \equiv 1(\bmod 4), \quad j=1, \ldots, n .
$$

If we note that $0 \leqq r_{j}, s_{j} \leqq n$, and $\sum_{j=1}^{2 n} r_{j}=\sum_{j=1}^{2 n} s_{j}=n$, then by using induction on $n$ we can show that the only solution is

$$
\mathbf{r}=(1, \ldots, 1,0, \ldots, 0), \quad \mathbf{s}=(0, \ldots, 0,1, \ldots, 1)
$$

where each vector has $n$ zeros and $n$ ones.

Thus (2.3) becomes $\sum_{\mathrm{k}, \mathrm{h}}(n !)^{2}=4^{2 n}(n !)^{2}$, and therefore (2.2) becomes $4^{2 n}(n !)^{2} x_{1}$ $\cdots x_{2 n}$. This completes the proof.

We have two immediate corollaries, the first of which was anticipated in the proof of Lemma (1.9).

COROllaRY (2.4). If $U$ is a linear operator from a $G_{2 n}$ space $X_{1}$ (with g.i.p. $\langle\cdot, \ldots, \cdot\rangle_{1}$ ) to a $G_{2 n}$ space $X_{2}$ (with g.i.p. $\langle\cdot, \ldots, \cdot\rangle_{2}$ ) then $U$ is an isometry if and only if

$$
\left\langle U\left(x_{1}\right), \ldots, U\left(x_{2 n}\right)\right\rangle_{2}=\left\langle x_{1}, \ldots, x_{2 n}\right\rangle_{1}
$$

for all $x_{1}, \ldots, x_{2 n}$ in $X_{1}$.

We shall return to isometries again in $\$ 4$.

CoROllary (2.5). If $X$ is a $G_{2 n}$ space then the g.i.p. is a continuous map from $X^{2 n}$, with the product topology, into the complex numbers.

The existence of the polarization identity leads one to consider the existence of the "parallelogram law for $G_{2 n}$ spaces." We first need some notation.

$$
\left\|x_{1} \pm \cdots \pm x_{p}\right\|^{2 n}=\sum_{n_{2}=0}^{1} \cdots \sum_{n_{p}=0}^{1}\left\|x_{1}+(-1)^{n_{2}} x_{2}+\cdots+(-1)^{n_{p}} x_{p}\right\|^{2 n}
$$

THEOREM (2.6). If $X$ is a $G_{2 n}$ space then

$$
\sum_{k=1}^{n+1}(-2)^{n+1-k} \sum_{1 \leqq j_{1}<\cdots<j_{k} \leqq n+1}\left\|x_{j_{1}} \pm \cdots \pm x_{j_{k}}\right\|^{2 n}=0
$$

where the second sum is to be taken over all the $C_{n+1, k}$ possible ways to pick the $k$ distinct integers $j_{1}, \ldots, j_{k}$ from the first $(n+1)$ integers.

In the case $n=1$ we get

$$
(-2)\left(\left\|x_{1}\right\|^{2}+\left\|x_{2}\right\|^{2}\right)+\left(\left\|x_{1}+x_{2}\right\|^{2}+\left\|x_{1}-x_{2}\right\|^{2}\right)=0,
$$

which is the parallelogram law for inner product spaces. 
Proof. The proof is somewhat like the proof of the last theorem and we shall suppress some of the details. First one shows for a fixed $j_{1}, \ldots, j_{k}$ that

$$
\left\|x_{j_{1}} \pm \cdots \pm x_{j_{k}}\right\|^{2 n}
$$

consists of terms of the form

$$
C_{n, \mathbf{r}} C_{n, \mathbf{s}} 2^{k-1} x_{j_{1}}^{r_{1}} \cdots x_{j_{k}}^{r_{k}} X_{j_{1}}^{s_{1}} \cdots x_{j_{k}}^{s_{k}}
$$

where $r_{i}+s_{i} \equiv 0(\bmod 2)$ for $i=1, \ldots, k$. Thus the most general term on the left hand side of (2.7) is

$$
A x_{t_{1}}^{a_{1}} \cdots x_{t_{m}}^{a_{m}} x_{t_{1}}^{b_{1}} \cdots x_{t_{m}}^{b_{m}}
$$

where we can assume that $a_{\mathfrak{i}}+b_{i}$ is even and not 0 for $i=1, \ldots, m$. The proof is completed by showing that $A=0$.

To see this we note that

$$
x_{t_{1}}^{a_{1}} \cdots x_{t_{m}}^{a_{m}} x_{t_{1}}^{b_{1}} \cdots x_{t_{m}}^{b_{m}}
$$

will appear in the sum (2.7) only when $k \geqq m$, and then only when $t_{1}, \ldots, t_{m}$ are among $j_{1}, \ldots, j_{k}$. This inclusion can happen in $C_{n+1-m, k-m}$ ways, and for each of these times (2.8) will appear $2^{k-1} C_{n, \mathbf{a}} C_{n, \mathrm{~b}}$ times. Thus the coefficient of (2.8) in (2.7) is

$$
\sum_{k=m}^{n+1}(-2)^{n+1-k} C_{n+1-m, k-m} 2^{k-1} C_{n, \mathbf{a}} C_{n, \mathbf{b}}=C_{n, \mathbf{a}} C_{n, \mathbf{b}} 2^{n}(1-1)^{n+1-k}=0 .
$$

We can now combine the last two results to obtain the Jordan-von Neumann theorem for $G_{2 n}$ spaces.

THeOREM (2.9). If $X$ is a normed linear space then $X$ is a $G_{2 n}$ space if and only if the norm satisfies the generalized parallelogram law (2.7).

Proof. The necessity follows from the last theorem. In order to establish the sufficiency we use Theorem (2.1) to provide us with the definition

$$
x_{1} \cdots x_{2 n}=4^{-2 n}(n !)^{-2} \sum_{\mathbf{h}, \mathbf{k}} i^{|\mathbf{h}|}\|F(\mathbf{x}, \mathbf{h}, \mathbf{k})\|^{2 n}
$$

We then note that we can write

$$
\sum_{\mathbf{h}, \mathbf{k}} i^{|\mathbf{h}|}\|F(\mathbf{x}, \mathbf{h}, \mathbf{k})\|^{2 n}=\sum_{\mathbf{h}, \mathbf{l}} i^{|\mathbf{h}|}\|G(\mathbf{x}, \mathbf{h}, \mathbf{l})\|^{2 n}
$$

where

$$
G(\mathbf{x}, \mathbf{h}, \mathbf{l})=i^{l_{1}}\left(x_{1}+i^{h_{1}} x_{n+1}\right) \pm \cdots \pm i^{l_{n}}\left(x_{n}+i^{h_{n}} x_{2 n}\right),
$$

$\mathbf{l}$ is an $n$-vector consisting of nonnegative integers either 0 or $1, \mathbf{h}$ is as before, and the sum is over all possible $\mathbf{h}$ and $\mathbf{l}$.

The proof now follows the same lines as the classical one [14, p. 124, Theorem 1] and the details will be omitted. 
In the development of the inner product space theory it is shown that the axioms for an inner product are sufficient to guarantee that it generates a norm. However, if in the definition of a g.i.p. we change axiom (i) to read

(i') $x^{2 n}>0$ if and only if $x \neq 0$,

then it does not follow that $\|x\|=\left(x^{2 n}\right)^{1 / 2 n}$ defines a norm. This is illustrated by the next example.

EXAMPLE (2.10). First observe that if $a$ and $b$ are complex numbers then $|a|^{4}$ $+16|a b|^{2}+|b|^{4}$ is generated by the 4 -form

$$
\begin{aligned}
x y z w=x_{1} y_{1} \bar{z}_{1} \bar{w}_{1}+4 x_{1} y_{2} \bar{z}_{1} \bar{w}_{2} & +4 x_{1} y_{2} \bar{z}_{2} \bar{w}_{1}+4 x_{2} y_{1} \bar{z}_{1} \bar{w}_{2} \\
& +4 x_{2} y_{1} \bar{z}_{2} \bar{w}_{1}+x_{2} y_{2} \bar{z}_{2} \bar{w}_{2} \quad\left(x=\left(x_{1}, x_{2}\right), \text { etc. }\right) .
\end{aligned}
$$

If we consider $X=C \times C$ as a vector space over $C$ (the complex numbers), then (2.11) satisfies axioms (i'), (ii), (iii), and (iv). However, if we let $x=(1,0)$ and $y=(0,1)$, we can see that $\|x\|=\left(x^{4}\right)^{1 / 4}=1,\|y\|=1$, and $\|x+y\|=18^{1 / 4}>2$. Hence the triangle inequality fails.

The question of necessary and sufficient conditions for $\|x\|=\left(x^{2 n}\right)^{1 / 2 n}$ to define a norm remains open.

3. CBS $G_{2 n}$ spaces. In light of example (2.10), it seems appropriate to consider a more restricted class of $G_{2 n}$ spaces called CBS $G_{2 n}$ spaces. These are spaces in which the g.i.p. satisfies the additional hypothesis

(v) $\left|x_{1} \cdots x_{2 n}\right| \leqq\left\|x_{1}\right\| \cdots\left\|x_{2 n}\right\|$ for all $x_{1}, \ldots, x_{2 n}$ in $X$.

All of the examples given so far are CBS $G_{2 n}$ spaces. However, the following modification of Example (2.10), illustrates that not all $G_{2 n}$ spaces are CBS $G_{2 n}$ spaces.

EXAmple (3.1). Again let $X=C \times C$ be considered as a vector space over $C$. Then $|a|^{4}+6|a b|^{2}+|b|^{4}$ is generated by the 4-form.

$$
\begin{aligned}
x y z w= & x_{1} y_{1} \bar{z}_{1} \bar{w}_{1}+\frac{3}{2} x_{1} y_{2} \bar{z}_{1} \bar{w}_{2}+\frac{3}{2} x_{1} y_{2} \bar{z}_{2} \bar{w}_{1} \\
& +\frac{3}{2} x_{2} y_{1} \bar{z}_{1} \bar{w}_{2}+\frac{3}{2} x_{2} y_{1} \bar{z}_{2} \bar{w}_{1}+x_{2} y_{2} \bar{z}_{2} \bar{w}_{2} .
\end{aligned}
$$

Then (3.2) satisfies axioms (i'), (ii), (iii), and (iv). It can be checked that if $\|x\|=\left(x^{4}\right)^{1 / 4}$ then $D=\{x: x \in X,\|x\| \leqq 1\}$ is absorbing, balanced, and convex [14, pp. 22-23]. (See Eggleston [1, p. 51] for a useful criterion for convexity.) Hence \|\| is a norm [10], and (3.2) is a g.i.p. On the other hand if we let $x=(1,0)$ and $y=(0,1)$, we have $\|x\|=\|y\|=1$ while

$$
x y x y=\frac{3}{2}>\|x\|^{2}\|y\|^{2} .
$$

The interest in CBS $G_{2 n}$ spaces is that in some respects they are very much like inner product spaces. As a result it is quite easy to extend some of the inner product space results and their proofs to this more general setting. For example, if a g.i.p. satisfies axioms (i'), and (ii)-(v), where $\|x\|=\left(x^{2 n}\right)^{1 / 2 n}$, then \|\| can be shown to be a norm by using the usual inner product space proof. 
Recall that a normed linear space is said to be uniformly convex if and only if given $\varepsilon>0$ there is a $\delta>0$ such that $\|x\| \leqq 1,\|y\| \leqq 1$, and $\|x+y\|>2-\delta$, implies $\|x-y\|<\varepsilon$.

Lemma (3.3). If $X$ is a $C B S G_{2 n}$ space then $X$ is uniformly convex.

Proof. Let $\|x\| \leqq 1$ and $\|y\| \leqq 1$. Then a straightforward calculation using the binomial expansion as a guide yields

$$
\begin{aligned}
\|x+y\|^{2 n}+\|x-y\|^{2 n} & =2 \sum_{\substack{k=0 \\
k+j \text { is even }}}^{n} \sum_{\substack{j=0 \\
n}}^{n} C_{n, k} C_{n, j} x^{k} y^{n-k} x^{j} y^{n-j} \\
& \leqq 2 \sum_{\substack{k=0 \\
k+j \text { is even }}}^{n} \sum_{j=0}^{n} C_{n, k} C_{n, j}=2^{2 n} .
\end{aligned}
$$

Thus $\|x-y\|^{2 n} \leqq 2^{2 n}-\|x+y\|^{2 n}$. Given $\varepsilon>0$ there is $\delta>0$ such that

$$
2^{2 n}-(2-\delta)^{2 n}<\varepsilon^{2 n} \text {. }
$$

Therefore $\|x+y\|>2-\delta$ implies $\|x-y\|^{2 n}<\varepsilon^{2 n}$.

By appealing to a theorem of Millman and Pettis [12] that states that a uniformly convex Banach space is reflexive, we can obtain the following corollary.

Corollary (3.4). If $X$ is a complete $C B S G_{2 n}$ space then $X$ is reflexive.

We shall refer to a complete $G_{2 n}$ space as an $H_{2 n}$ space.

It should be noted that if $X$ is a CBS $G_{2 n}$ space then

$$
\begin{aligned}
{[x, y] } & =\left(x y^{2 n-1}\right)\|y\|^{2-2 n} & & \text { if } y \neq 0, \\
& =0 & & \text { if } y=0
\end{aligned}
$$

defines a semi-inner-product. (See [9] for a discussion of semi-inner-products.) In particular a CBS $G_{2 n}$ space is an example of a continuous semi-inner-product space studied by Giles [4]. (See [6] for related results.) The remaining results can be easily obtained from Giles' work and so the proofs will be omitted. (Direct proofs in the present setting can easily be obtained by modifying the appropriate inner product space proofs.)

LEMMA (3.5). If $X$ is a CBS $G_{2 n}$ space then $x$ is orthogonal to $y(\|x\| \leqq\|x+\lambda y\|$ for all scalars $\lambda$ ) if and only if $y x^{2 n-1}=0[4$, p. 438, Theorem 2].

Lemma (3.6). If $X$ is a $C B S G_{2 n}$ space, $H$ is any complete subspace of $X$, and if $x$ is in $X$, then there is a unique $y$ in $H$ such that $z(x-y)^{2 n-1}=0$ for all $z$ in $H$ [4, p. 440, Lemma 4].

Theorem (3.7) (Generalized Riesz-Fréchet Representation TheOrem). Let $X$ be a CBS $H_{2 n}$ space and let $f$ be a continuous linear functional on $X$. Then there is a unique vector $y$ in $X$ such that $f(x)=x y^{2 n-1}$ for all $x$ in $X$, and $\|f\|=\|y\|^{2 n-1}$ [4, p. 441, Theorem 6]. 
4. Isometries on $G_{2 n}$ spaces. Fixmann [2, p. 1046, Theorem 5.2] has shown that there are invertible isometries on $l^{p}, p \neq 2$, that are not spectral. As a result, a spectral theorem for invertible isometries on $G_{2 n}$ spaces is not possible.

However, there is some spectral theory possible for isometries on an arbitrary complex normed linear space. (See [3], [7], [11], and [13].) And, in the finite dimensional $G_{2 n}$ case the analysis is complete.

First of all, it is known that every isometry of a finite dimensional complex normed linear space has a basis consisting of eigenvectors [7]. (A direct proof for the $G_{2 n}$ spaces is easy by using induction on the dimension and Corollary (2.4).) A type of converse is also known [11]. Namely, if an operator has a basis consisting of eigenvectors with eigenvalues on the unit circle, then it is an isometry in some equivalent norm. The next theorem is a more satisfactory converse for the $G_{2 n}$ spaces.

TheOREM (4.1). Let $X$ be a finite dimensional $G_{2 n}$ space, and suppose $U$ is an operator given by a diagonal matrix dia $\left(\lambda_{1}, \ldots, \lambda_{m}\right)$ relative to a basis $\left\{e_{1}, \ldots, e_{m}\right\}$. Then $U$ is an isometry if and only if

$$
\lambda_{1}^{r_{1}} \cdots \lambda_{m}^{r_{m}} \lambda_{1}^{s_{1}} \cdots \lambda_{m}^{s_{m}}=1 \quad \text { whenever } e_{1}^{r_{1}} \cdots e_{m}^{r_{m}} e_{1}^{s_{1}} \cdots e_{m}^{s_{m}} \neq 0
$$

where $r_{1}+\cdots+r_{m}=s_{1}+\cdots+s_{m}=n$.

It should be noted that in the case of the inner product space the condition becomes

$$
\lambda_{i} \lambda_{j}=1 \text { whenever } e_{i} e_{j} \neq 0 .
$$

And if $\left\{e_{1}, \ldots, e_{m}\right\}$ is an orthonormal basis, the condition is merely $\left|\lambda_{i}\right|^{2}=1$, $i=1, \ldots, m$.

Proof. If $U$ is an isometry then by Corollary (2.4), $U$ preserves the g.i.p. As a result we have

$$
\begin{aligned}
e_{1}^{r_{1}} \cdots e_{m}^{r_{m}} e_{1}^{s_{1}} \cdots e_{m}^{s_{m}} & =\left(U\left(e_{1}\right)\right)^{r_{1}} \cdots\left(U\left(e_{m}\right)\right)^{r_{m}}\left(U\left(e_{1}\right)\right)^{s_{1}} \cdots\left(U\left(e_{m}\right)\right)^{s_{m}} \\
& =\lambda_{1}^{r_{1}} \cdots \lambda_{m}^{r_{m}} \lambda_{1}^{s_{1}} \cdots \lambda_{m}^{s_{m}} e_{1}^{r_{1}} \cdots e_{m}^{r_{m}} e_{1}^{s_{1}} \cdots e_{m}^{s_{m}} .
\end{aligned}
$$

Therefore the condition is necessary.

To see that the condition is sufficient we need to establish that

$$
\left\|U\left(\sum x_{j} e_{j}\right)\right\|^{2 n}=\left\|\sum x_{j} e_{j}\right\|^{2 n} \text {. }
$$

But

$$
\left\|U\left(\sum x_{j} e_{j}\right)\right\|^{2 n}=\left\|\sum \lambda_{j} x_{j} e_{j}\right\|^{2 n}=\sum_{\mathbf{r}, \mathbf{s}} c(\lambda \mathbf{x}, \mathbf{r}, \mathbf{s}) d(\mathbf{e}, \mathbf{r}, \mathbf{s})
$$

where

$$
c(\lambda \mathbf{x}, \mathbf{r}, \mathbf{s})=C_{n, \mathbf{r}} C_{n, \mathbf{s}}\left(\lambda_{1} x_{1}\right)^{r_{1}} \cdots\left(\lambda_{m} x_{m}\right)^{r_{m}}\left(\bar{\lambda}_{1} \bar{x}_{1}\right)^{s_{1}} \cdots\left(\bar{\lambda}_{m} \bar{x}_{m}\right)^{s_{m}}
$$


Because of condition (4.2) we have that whenever

$$
\begin{aligned}
d(\mathbf{e}, \mathbf{r}, \mathbf{s}) & =e_{1}^{r_{1}} \cdots e_{m}^{r_{m}} e_{1}^{s_{1}} \cdots e_{m}^{s_{m}} \neq 0 \\
(4.3) & =C_{n, \mathbf{r}} C_{n, \mathbf{s}} x_{1}^{r_{1}} \cdots x_{m}^{r_{m}} \bar{x}_{1}^{s_{1}} \cdots \bar{x}_{m}^{s_{m}}=c(\mathbf{x}, \mathbf{r}, \mathbf{s}) .
\end{aligned}
$$

Therefore

$$
\sum_{\mathbf{r}, \mathbf{s}} c(\lambda \mathbf{x}, \mathbf{r}, \mathbf{s}) d(\mathbf{e}, \mathbf{r}, \mathbf{s})=\sum_{\mathbf{r}, \mathbf{s}} c(\mathbf{x}, \mathbf{r}, \mathbf{s}) d(\mathbf{e}, \mathbf{r}, \mathbf{s})=\left\|\sum_{j} x_{j} e_{j}\right\|^{2 n}
$$

Equivalently the theorem requires that

$$
e_{1}^{r_{1}} \cdots e_{m}^{r_{m}} e_{1}^{s_{1}} \cdots e_{m}^{s_{m}}=0 \quad \text { whenever } \lambda_{1}^{r_{1}} \cdots \lambda_{m}^{r_{m}} \lambda_{1}^{s_{1}} \cdots \lambda_{m}^{s_{m}} \neq 1 .
$$

These are all types of "orthogonality" that are required in order for a $G_{2 n}$ space to have nontrivial isometries. It is possible that a $G_{2 n}$ space with "limited types of orthogonality" may have no nontrivial isometries.

5. Symmetric operators on CBS $H_{2 n}$ spaces. We now turn to a study of "symmetric" operators on CBS $H_{2 n}$ spaces and explore the relationship between them and the geometry of the space.

Definition (5.1). Let $X$ be a CBS $H_{2 n}$ space and $T$ be an operator (not necessarily linear) from $X$ into itself. We shall say that $T$ is symmetric if and only if

$$
x_{1} \cdots T\left(x_{i}\right) \cdots x_{2 n}=x_{1} \cdots T\left(x_{j}\right) \cdots x_{2 n}
$$

for all $i$ and $j$, and all $x_{1}, \ldots, x_{2 n}$ in $X$.

With a little modification the usual Hilbert space proofs for selfadjoint operators provide us with the following facts.

LeMma (5.2). If $T$ is symmetric then:

(i) $T$ is linear and continuous.

(ii) Any real polynomial in $T$ is symmetric.

(iii) The spectrum of $T$ is real.

(iv) $\|T\|=r(T)$ where $r(T)$ is the spectral radius of $T$.

With the help of this lemma we can see that if we have a given symmetric operator $T$, then there is an isometric isomorphism between the algebra of polynomials in $T$ with the operator norm and the algebra of polynomials restricted to the spectrum of $T$ with the sup norm. But this is sufficient to establish a spectral theorem [13, p. 507, Theorem 2].

THEOREM (5.3). If $X$ is a CBS $H_{2 n}$ space and $T$ is a symmetric operator on $X$, then there is a unique symmetric-projection-valued spectral measure $E$ on the Borel sets of $\sigma(T)$ such that $T=\int_{\sigma(T)} \lambda d E(\lambda)$.

Proof. We only need to establish that the projections are symmetric. But this follows since the projections are the weak operator limits of real polynomials in $T$ (which are symmetric), and such limits of symmetric operators are again symmetric. 
Thus the existence of symmetric operators is equivalent to the existence of symmetric projections, and we conclude our study with a few remarks about symmetric projections.

TheOREM (5.4). Let $X$ be a $G_{2 n}$ space. Then $X$ is isometrically isomorphic to the direct sum of two nontrivial $G_{2 n}$ spaces $Y_{1}$ and $Y_{2}$ if and only if there is a symmetric projection $P$ defined on $X$ such that $P(X)$ is isometrically isomorphic to $Y_{1}$ and $(I-P)(X)$ is isometrically isomorphic to $Y_{2}$.

Proof. If a symmetric projection exists then $X=P(X)+(I-P)(X)$. We need only show that the norm on $X$ is the same as the norm on $Y_{1} \oplus Y_{2}$ generated by (1.10). So let $Q=I-P$. Then

$$
\|x\|^{2 n}=(x)^{2 n}=(P(x)+Q(x))^{2 n}=\sum_{k, j} C_{n, k} C_{n, j}(P(x))^{k}(Q(x))^{n-k}(P(x))^{j}(Q(x))^{n-j} .
$$

Since $P$ and $Q$ are symmetric and $P Q=Q P=0$ the only terms to survive are

$$
C_{n, n} C_{n, n}(P(x))^{2 n}+C_{n, 0} C_{n, 0}(Q(x))^{2 n}=(P(x))^{2 n}+(Q(x))^{2 n} \equiv\|(P(x), Q(x))\|^{2 n} .
$$

Conversely, suppose $X$ is isometrically isomorphic to $Y_{1} \oplus Y_{2}$, considered as the direct sum of two $G_{2 n}$ spaces. Let $X_{1}$ and $X_{2}$ be the images of $Y_{1} \oplus\{0\}$ and $\{0\} \oplus Y_{2}$ respectively. Then $X=X_{1}+X_{2}$. Let $P$ be the natural projection of $X$ on $X_{1}$ along $X_{2}$. Then for $x_{1}, \ldots, x_{2 n}$ in $X\left(x_{i}=y_{i}+z_{i}, y_{i} \in X_{1}, z_{i} \in X_{2}\right)$, we have

$$
\begin{aligned}
P\left(x_{1}\right) \cdots x_{2 n} & =\left\langle\left(y_{1}, 0\right), \ldots,\left(y_{2 n}, z_{2 n}\right)\right\rangle \\
& =\left\langle y_{1}, \ldots, y_{2 n}\right\rangle_{1}+\left\langle 0, \ldots, z_{2 n}\right\rangle_{2} \\
& =\left\langle y_{1}, \ldots, y_{2 n}\right\rangle_{1}+\left\langle z_{1}, \ldots, z_{i-1}, 0, z_{i+1}, \ldots, z_{2 n}\right\rangle_{2} \\
& =\left\langle\left(y_{1}, z_{1}\right), \ldots,\left(y_{i}, 0\right), \ldots,\left(y_{2 n}, z_{2 n}\right)\right\rangle \\
& =x_{1} \cdots P\left(x_{i}\right) \cdots x_{2 n} .
\end{aligned}
$$

This is sufficient to show that $P$ is symmetric.

This theorem illustrates the connection between symmetric operators and the geometry of the spaces. It is interesting to consider some of our examples in light of these results. For example, if $P$ is a nontrivial symmetric operator and $Q=I-P$, then we have noted that

$$
(P(x))(Q(x))^{n-1}(P(x))(Q(x))^{n-1}=0
$$

for all $x$. But this condition is not possible in either Example (1.4) or Example (1.6). As a result neither of these spaces have any nontrivial symmetric operators. Further, because of the last theorem we can see that those spaces with the "largest" number of symmetric operators will be those spaces that are isometrically isomorphic to the direct sum of one dimensional CBS $H_{2 n}$ spaces, and thus to $l^{2 n}$ of some set. What is needed is to find other classes of operators associated with the various types of "non- $l^{2 n}$ " CBS $H_{2 n}$ spaces. 


\section{REFERENCES}

1. H. G. Eggleston, Convexity, Cambridge Tracts in Math. and Math. Phys., no. 47, Cambridge Univ. Press, New York, 1958. MR 23 \#A2123.

2. U. Fixmann, Problems in spectral operators, Pacific J. Math. 9 (1959), 1029-1051. MR 21 \#7441.

3. S. R. Foguel, The relations between a spectral operator and its scalar part, Pacific J. Math. 8 (1958), 51-65. MR 20 \#3457.

4. J. R. Giles, Classes of semi-inner-product spaces, Trans. Amer. Math. Soc. 129 (1967), 436-446. MR 36 \#663.

5. P. Jordan and J. von Neumann, On inner products in linear metric spaces, Ann. of Math. (2) 36 (1935), 719-723.

6. D. O. Koehler, A note on some operator theory in certain semi-inner-product spaces, (to appear).

7. D. O. Koehler and Peter Rosenthal, On isometries of normed linear spaces, Studia Math. 36 (1970), 215-218.

8. L. H. Loomis, An introduction to abstract harmonic analysis, Van Nostrand, Princeton, N. J., 1953. MR 14, 883.

9. G. Lumer, Semi-inner-product spaces, Trans. Amer. Math. Soc. 100 (1961), 29-43. MR 24 \#A2860.

10. H. Minkowski, “Theorie der Konvexen Körper, insbesondere Begründung ihres Oberflächenbegriffs," in Ges Abhandl. Vol. 2, Leipzig-Berlin, 1911, pp. 131-229.

11. T. V. Panchapagesan, Unitary operators in Banach spaces, Pacific J. Math. 22 (1967), 465-475. MR 35 \#4762.

12. B. J. Pettis, A proof that every uniformly convex space is reflexive, Duke Math. J. 5 (1939), 249-253.

13. J. G. Stampfli, Adjoint abelian operators on Banach space, Canad. J. Math. 2 (1969), 505-512.

14. A. Wilansky, Functional analysis, Blaisdell, Waltham, Mass., 1964. MR $30 \# 425$.

\section{MIAMI UNIVERSITY,}

OXFORD, ОHIO 45056 